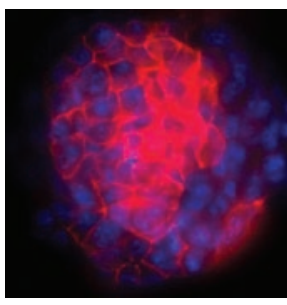

\title{
Progress in tumor progression
}

Activation of the transcription factor NF-KB occurs in many human tumors, and studies have shown that NF$\kappa \mathrm{B}$ can promote cell proliferation and oncogenesis, possibly by protecting cells from apoptosis. Little is known, however, about whether NF- $\mathrm{KB}$ is involved in tumor progression. Thomas Wirth and colleagues used an in vitro/in vivo model of mammary carcinogenesis to investigate the involvement of NF- $\kappa B$ in epithelial plasticity and metastasis (pages 569-581). The authors showed that NF- $\mathrm{KB}$ is essential for epithelial-mesenchymal transition (EMT), which is thought to be important for tumor invasion. Ha-Ras-transformed mammary epithelial cells, which undergo EMT in response to TGF- $\beta$, were prevented from undergoing EMT when NF- $\mathrm{KB}$ was

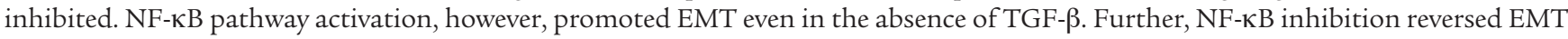
in mesenchymal cells. Blocking the NF-KB pathway in nude mice injected with Ha-Ras-transformed mammary epithelial cells abolished the metastatic potential of these cells. Taken together, these data provide evidence for an essential role of NF-KB in the induction and maintenance of EMT and mark the NF-KB pathway as a potential anti-metastatic therapeutic target.

\section{Having an affinity for diabetes}

Insulin autoantibodies (IAAs) are often the first autoantibody recognized in the natural history of childhood diabetes. Not all IAA-positive children, however, go on to develop additional autoantibodies against antigens from pancreas islet cells and, from there, progress to type 1 diabetes mellitus (T1DM). In a standard immune response, antibody maturation through repeated exposure to antigen results in increased antibody affinity. Ezio Bonifacio and colleagues analyzed IAA affinity in IAA-positive samples from children of the BABYDIAB cohort to examine the relationship of antibody affinity to development of T1DM (pages 589-597). Using a competitive radiobinding assay, the authors found that the presence of high-affinity IAAs in a sample correlated with the individual having HLA DRB $1 * 04$ and subsequently developing multiple islet autoantibodies and T1DM. In examining epitope specificity, they further found that high- and low-affinity IAAs were reactive with different insulin epitopes and that high-affinity IAAs were reactive with (pro)insulin, while low-affinity antibodies were not. The findings here indicate that early exposure to (pro)insulin may be important for disease pathology and that relative IAA affinity and epitope reactivity may be useful in classifying islet cell autoimmunity stages and establishing diabetes risk.

\section{Collecting duct collects ET-1 data}

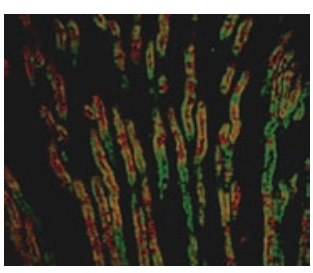

Endothelin-1 (ET-1) is thought to play an important role in regulating $\mathrm{Na}$ reabsorption. Many of the biological details of ET-1 activity have been defined in vitro, while conclusive physiological data from in vivo studies have been limited by the lethality of ET-1knockout mice and by an inability to discriminate between ET-1 effects in the nephron and those in the vasculature in conditional mutants. Donald Kohan and colleagues have now created a mouse model in which ET-1 is selectively disrupted in the collecting duct (CD) and investigated its physiological importance (pages 504-511). These mice, when fed a normal $\mathrm{Na}$ diet, were hypertensive, but their body weight, $\mathrm{Na}$ excretion, urinary aldosterone excretion and plasma renin activity were similar to those in wild-type mice. When the mice were given a high-Na diet, hypertension worsened, but they additionally had excessive weight gain and reduced $\mathrm{Na}$ excretion, which indicates that ET-1 affects salt retention under Na-loading conditions. Treatment of normal- or high-Na-diet mice with the natriuretic agents amiloride or furosemide reduced blood pressure and alleviated $\mathrm{Na}$ retention, which suggests that $\mathrm{CD}$-derived ET-1 regulates $\mathrm{Na}$ reabsorption through inhibition of tubule Na reabsorption. These data define an important physiological role for CD-derived ET-1 in regulating systemic blood pressure and renal $\mathrm{Na}$ excretion.

\section{MICs shed light on prostate cancer}

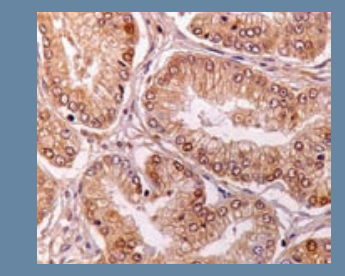

MHC class I chain-related molecule (MIC) triggers NK cell anti-tumor activity via engagement to its receptor NKG2D. The MIC-NKG2D system is known to be involved in epithelial tumor immune surveillance. Jennifer $\mathrm{Wu}$ and colleagues now investigate a role for the MIC-NKG2D system in prostate cancer (pages 560-568). The researchers found that MIC-expressing prostate cancer cell lines were susceptible to NK activation. Analysis of prostate tumor biopsies showed that surface localization of MIC was highest in early-stage tumors. High levels of serum soluble MIC (sMIC) and deficiency in NK cell activation were found in later-stage tumor patients, which indicates that MIC shedding is a means by which prostate cancer cells could overcome the MIC-NKG2D immune surveillance. While sMIC serum levels did not correspond with prostate-specific antigen serum levels, they were highly correlative with highgrade and invasive tumor status in prostate cancer patients. The deficiency in NK cell activation could be overcome in vitro by stimulation with IL-2 or IL-15. This work indicates that development of the means to evade the MIC-NKG2D system may be a mechanism for prostate cancer progression, that sMIC may be a useful biomarker for disease progression, and that cytokine treatment may aid in reestablishing NK cell anti-tumor activity. 